\title{
Brain-Stem Perturbations During Cortically Evoked Rhythmical Jaw Movements: Effects of Activation of Brain-Stem Loci on Jaw Muscle Cycle Characteristics
}

\author{
Scott H. Chandler and Michael Tala \\ Department of Kinesiology and the Brain Research Institute, University of California, Los Angeles, Los Angeles, California \\ 90024
}

The purpose of the present study was to further elucidate with the use of microstimulation techniques the influences of rostral pons and midbrain loci on the neuronal networks responsible for cortically induced rhythmical jaw movement (RJM) activity in the anesthetized guinea pig and to establish if these rostral brain-stem loci are capable of modulating the timing as well as the amplitude of rhythmical digastric (DIG) EMG activity. It was found that repetitive electrical stimulation of widespread areas of the rostral pons and midbrain produced suppression of ongoing cortically induced rhythmical EMG activity. Prior to complete EMG suppression there was a reduction in amplitude of the DIG EMG and an increase in cycle duration. Repetitive stimulation of these suppressive loci also produced a reduction in the amplitude of the short-latency DIG EMG response produced by short pulse train stimulation of the masticatory cortex. This indicates that part of the suppression of cortically induced rhythmical EMG activity was due to a reduction in excitability of the polysynaptic short-latency pathway from cortex to DIG motoneurons. Short pulse train stimulation of these brain-stem suppressive loci during various phases of the rhythmical DIG cycle produced a phase-dependent increase in duration of the ongoing perturbed cycle. The cycles following the stimulus perturbation did not show any compensatory shortening in their durations suggesting that the stimulus produced a true resetting of the cycle. These data suggest that the brain-stem loci that produce suppression of RJMs evoked by repetitive cortical stimulation can affect the excitability of the central circuits responsible for cycle oscillation and timing as well as the excitability of the polysynaptic short-latency corticotrigeminal pathway to DIG motoneurons.

The reticular formation of the brain stem has been implicated in the control of motor behaviors such as locomotion (Grillner, 1981, for review), eye movements (Robinson, 1981, for review),

Received Feb. 28, 1986; revised June 16, 1986; accepted July 14, 1986.

This research was supported by the National Institute of Dental Research Grant DE 06193. We would like to thank Naomi Goffman for her excellent histological assistance and Leon Salem for assisting in data collection. We would also like to thank the anonymous reviewers for their excellent suggestions for improvement of this manuscript.

Correspondence should be addressed to Scott $\mathrm{H}$. Chandler, $\mathrm{Ph}$.D., Department of Kinesiology, UCLA, 2851 Slichter Hall, 405 Hilgard Avenue, Los Angeles, CA 90024.

a Dr. Tal was on sabbatical leave from the Hebrew University, Department of Anatomy, School of Dentistry, Jerusalem, Israel.

Copyright (C 1987 Society for Neuroscience $0270-6474 / 87 / 020463-10 \$ 02.00 / 0$ respiration (Wyman, 1977; Von Euler, 1983; Feldman, 1986, for reviews), and jaw movements (Dubner et al., 1978; Nakamura et al., 1979; Luschei and Goldberg, 1981, for reviews) among others (reviewed in Siegal, 1979). Furthermore, particular reticular loci of the brain stem were shown to be involved with the control of pain suppression (Basbaum and Fields, 1978, for review) and muscular activity during sleep (Steriade and Hobson, 1976, for review).

With respect to the control of jaw muscle activity, numerous studies have investigated the role of supraspinal brain-stem mechanisms controlling jaw movements evoked by reflex activation (Dubner et al., 1978; Luschei and Goldberg, 1981, for review). In particular, widespread areas of the brain stem were shown to produce suppression of the jaw opening reflex (JOR) evoked by noxious and non-noxious oral facial receptor activation (Oliveras et al., 1974; Sessle and $\mathrm{Hu}, 1981$; Sessle et al., 198 la, b; Dostrovsky et al., 1982; Hayashi et al., 1984). There are also a number of studies that focused on determining the elements within the bulbar reticular formation that comprise the particular components of the central neuronal networks responsible for rhythmical jaw movements resembling mastication and other rhythmical oral motor behaviors in various animal species (Nakamura et al., 1975, 1979, 1980 Takatori et al., 1981; Nozaki et al., 1983, 1986; Marini and Sotgiu, 1985; Chandler and Tal, 1986). Electrophysiological (Nakamura et al., 1975; Nozaki et al., 1983) and anatomical (Mizuno et al., 1983; Travers and Norgren, 1983; Vornov and Sutin, 1983) studies have identified trigeminal pre-motoneurons within the lower brainstem reticular formation. Furthermore, neurons within this region were shown to be rhythmically active during food ingestion (Nakamura et al., 1982, 1984).

There are few studies, however, that examined the ability of brain-stem loci to modulate ongoing RJM activity. Sumi (1971) showed that electrical stimulation of widespread areas of the brain-stem reticular formation produced suppression of ongoing EMG activity and RJMs. An important question that has not been addressed is whether these loci are capable of modulating the frequency and timing of jaw muscle activity as well as the amplitude and duration of EMG activity. In the early studies on the supraspinal control of locomotion, it was proposed that brain-stem loci control only the level of motoneuron excitability, while central timing of muscle synergy was controlled by higher centers (Orlovsky, 1972). Subsequently, it was shown that brain-stem loci were capable of modulating the central ncuronal circuits responsible for oscillation and timing (Russell and Zajac, 1979; Drew and Rossignol, 1984). Furthermore, we 
have shown that pontomedullary reticular formation sites are capable of controlling the central networks responsible for oscillation during RJMs in the guinea pig (Chandler and Goldberg, 1986).

In recent studies, it was shown with lesion and transection techniques that brain-stem loci rostral to the motor nucleus of $\mathrm{V}$ (excluding pyramidal fibers) are not essential for RJM activity induced by cortical stimulation (Chandler and Goldberg, 1984; Chandler and Tal, 1986; Nozaki et al., 1986). This, of course, does not exclude these regions from functioning as initiators or modulators of RJM behavior. In fact, we recently showed that RJMs induced by dopaminergic activation are dependent on the integrity of the superior colliculus and surrounding reticular formation (Chandler and Goldberg, 1984).

The purpose of the present study was to further elucidate with the use of microstimulation techniques the influences of rostral pons and midbrain suppressive loci on the neuronal networks responsible for cortically induced RJM activity in the anesthetized guinea pig and to establish if these rostral brain-stem loci are capable of modulating the timing of rhythmical EMG activity as well as its amplitude and duration.

\section{Materials and Methods}

The following experiments were performed on 15 male, albino guinea pigs weighing $400-600 \mathrm{gm}$. Thirty minutes before administration of the anesthetic agent, the animals were given Thorazine (chlorpromazine hydrochloride, $12.5 \mathrm{mg} / \mathrm{kg}, i . \mathrm{m}$.) and atropine sulfate $(0.1 \mathrm{mg} / \mathrm{kg}$, i.p.). The animals then were anesthetized with ketamine $\mathrm{HCl}(110 \mathrm{mg} / \mathrm{kg}$, i.m.). A midline incision was made from the mental symphysis on the inferior surface of the mandible to the sternum. The trachea and external jugular vein were cannulated and the anterior belly of the digastric (DIG) muscle on both sides of the mandible was exposed for insertion of bipolar electromyographic (EMG) recording electrodes (0.13 mm O.D., tip exposure $2.0 \mathrm{~mm}$ ). In addition, EMG recording electrodes were inserted into the superficial and deep masseter muscles bilaterally. This procedure was not carried out in all experiments since these muscles are seldom active during cortically evoked RJMs, and in the initial experiments of this study it was found that brain-stem stimulation of loci which suppressed RJMs did not affect the background activity of these muscles. The animals were placed in a Kopf stereotaxic device and a circular piece of skull ( $8 \mathrm{~mm}$ in diameter, $2 \mathrm{~mm}$ anterior to bregma and $3 \mathrm{~mm}$ lateral to the midline) overlying the right masticatory cortex was removed. A bipolar strut stimulating electrode was inserted into the cortex to induce rhythmical digastric activity (RDA) and the corresponding RJMs. Previous experiments from our laboratory have shown an excellent correspondence between rhythmical DIG EMG activity and jaw opening movements (Goldberg et al., 1982). Therefore, in these experiments jaw movements were not monitored. Cortical stimulation consisted of continuous repetitive pulses at $40 \mathrm{~Hz}, 0.5 \mathrm{msec} / \mathrm{pulse}$, at 1.5-2 times threshold for inducing RDA (thresholds ranged from 50 to $200 \mu \mathrm{A}$ ). In some cases, the cortex was stimulated with short pulse trains (SPT; 1/sec, $500 \mathrm{~Hz}, 3-4$ pulses, $0.5 \mathrm{msec} /$ pulse) to initiate a shortlatency DIG EMG response without RJMs. The intensity of stimulation was adjusted to approximately twice threshold for the visible initiation of the response in the contralateral DIG EMG (thresholds ranged from 80 to $300 \mu \mathrm{A}$ ). The amplitude produced by this stimulation was between 50 and $80 \%$ of the maximum peak amplitude that could be obtained. The identification of the masticatory cortex in the guinea pig was described in detail elsewhere (Goldberg et al., 1982). Supplemental doses of ketamine (25-50 mg, i.v.) were given every $2 \mathrm{hr}$ or if any signs of spontaneous limb movements were present. Under these conditions the animal was not responsive to pinching of the pad of either foot or pinna and would lie motionless during interstimulus trials.

To investigate the effects of stimulation of midbrain and rostral pontine loci on cortically induced RDA, the left occipital cortex was removed by aspiration thereby exposing the surface of the tectum. In some experiments the rostral third of the cerebellum was removed to expose the floor of the 4th ventricle and underlying pontine brain stem. A bipolar concentric, semi-microelectrode (Kopf MCE-100, tip exposure $25 \mu \mathrm{m}$, indifferent surface exposure $100 \mu \mathrm{m}$ (shaft diameter 150 $\mu \mathrm{m})$, interelectrode distance $100 \mu \mathrm{m}$ ) was inserted into the brain stem at an angle of $16^{\circ}$ from the vertical in the caudal to rostral direction. This produced electrode penetrations that were perpendicular to the brain-stem surface. Histological sections were cut in the plane of the electrode. From 10 animals the total brain-stem area examined was a region bounded in the rostral-caudal plane by the substantia nigra rostrally and extending to the rostral boundary of the trigeminal motor nucleus caudally. The medial-lateral plane extended from midline to $2.5 \mathrm{~mm}$ lateral to midline. In any particular experiment only a small defined region of brain stem was explored in increments of $0.5 \mathrm{~mm}$ in the rostral-caudal (maximum of $2 \mathrm{~mm}$ ), medial-lateral, and dorsalventral directions. A maximum of 10 electrode penetrations was made in an experiment.

To determine the effects of electrical stimulation of different loci on RDA, the stimulus to the brain stem consisted of a continuous train of stimuli at $50 \mathrm{~Hz}(0.15 \mathrm{msec}$ pulse duration) for 8-12 sec during cortically induced RDA. The maximum stimulus intensity examined for quantification of brain-stem effects was $50 \mu \mathrm{A}$. Intensities greater than this produced other body movements such as rhythmical fore and hindlimb movements, escapelike responses, and whole-body jerks. The threshold for complete cessation of RDA activity within $1 \mathrm{sec}$ of the onset of the brain-stem stimulus most often ranged between 20 and $50 \mu \mathrm{A}$ but sometimes was as low as $15 \mu \mathrm{A}$. In the ketamine-anesthetized preparation these values would vary depending on the level of anesthesia during the course of the experiment. Therefore, it was not possible to construct accurate isothreshold curves for the various brain-stem regions. Loci were considered effective if upon activation they consistently produced complete cessation of RDA within $1 \mathrm{sec}$ of onset of the brain-stem stimulus at less than $50 \mu \mathrm{A}$. With the variability observed using these methods it was not possible to differentiate the effects on RDA between closely adjacent reticular formation loci. Nevertheless, using these criteria, the general trend (facilitation, suppression, or no effect) of effects of brain-stem activation on RDA were obtained. In addition, the effects of the brain-stem stimulus on the short-latency DIG response to SPT stimulation of the cortex were determined. The cortex was stimulated with a short pulse train at $1 / \mathrm{sec}(500 \mathrm{~Hz}, 3-4$ pulses, $0.5 \mathrm{msec})$ and the subsequent DIG EMG activity was averaged with a 4 channel Dagan signal averager. The mean peak amplitudes prior to and during repetitive brain-stem stimulation were compared. A minimum of 16 consecutive responses for test and control conditions was obtained. At the termination of an experiment, 3 small electrolytic lesions were placed in the brain stem. One lesion corresponded to a site that produced the lowest threshold effects; another lesion was placed either 1 or $2 \mathrm{~mm}$ above or below that lesion in the same anterior-posterior plane, while a third was placed at the same depth of one of the previous lesions either 1 $\mathrm{mm}$ rostral or caudal to those lesions. The effective sites were then reconstructed histologically with reference to the electrolytic lesions and related to the stereotaxic atlas of the guinea pig by Rossner (1965) and nomenclature used by Petrovicky (1966).

In another series of experiments $(n=5)$, the effects of short pulse train stimulation of brain-stem sites on the cortically induced RDA cycle characteristics [cycle duration (CD), burst duration (BD), integrated EMG (I-EMG)/BD] were examined. The sites chosen were those producing complete suppression of RDA during repetitive stimulation at less than $50 \mu \mathrm{A}$. In any particular experiment usually data from 1 or 2 sites were recorded on tape for subsequent quantitative analyses. To quantify changes in EMG amplitude, the value of the mean I-EMG divided by mean $\mathrm{BD}$ was determined (I-EMG/BD). This normalization procedure allows one to quantify mean changes in amplitude irrespective of changes in duration of the bursts. A short pulse train stimulus $(50-100 \mathrm{~Hz}, 50-400 \mathrm{msec}, 25-50 \mu \mathrm{A}, 0.15 \mathrm{msec})$ was elicited at various phases of the RDA cycle. The phase of the cycle the stimulus was elicited was defined as the time from DIG burst onset to the onset of the stimulus train divided by the duration of the preceding RDA cycle ( $P-1$ cycle). The phase of the cycle the stimulus was delivered is expressed as a percentage of the mean control $\mathrm{CD}$ and has a range from 0 to 1 . The onset of a DIG EMG burst determined the onset of an RDA cycle (Fig. $5 A$ ), while the cycle the stimulus was elicited is termed the perturbed cycle (Fig. $5 A, \mathrm{P}$ cycle). The subsequent cycle after the perturbed cycle is termed the $P+1$ cycle, the cycle after that is termed the $P+2$ cycle, etc. (Fig. $5 A$ ). The cycle immediately prior to the perturbed cycle is termed the $\mathrm{P}-1$ cycle (Fig. $5 A$ ). This cycle served as the control cycle with respect to the subsequent cycles. The effect of the stimulus on the duration of the perturbed cycle and subsequent cycle characteristics were examined. Such analyses allowed us to determine if these loci, when 

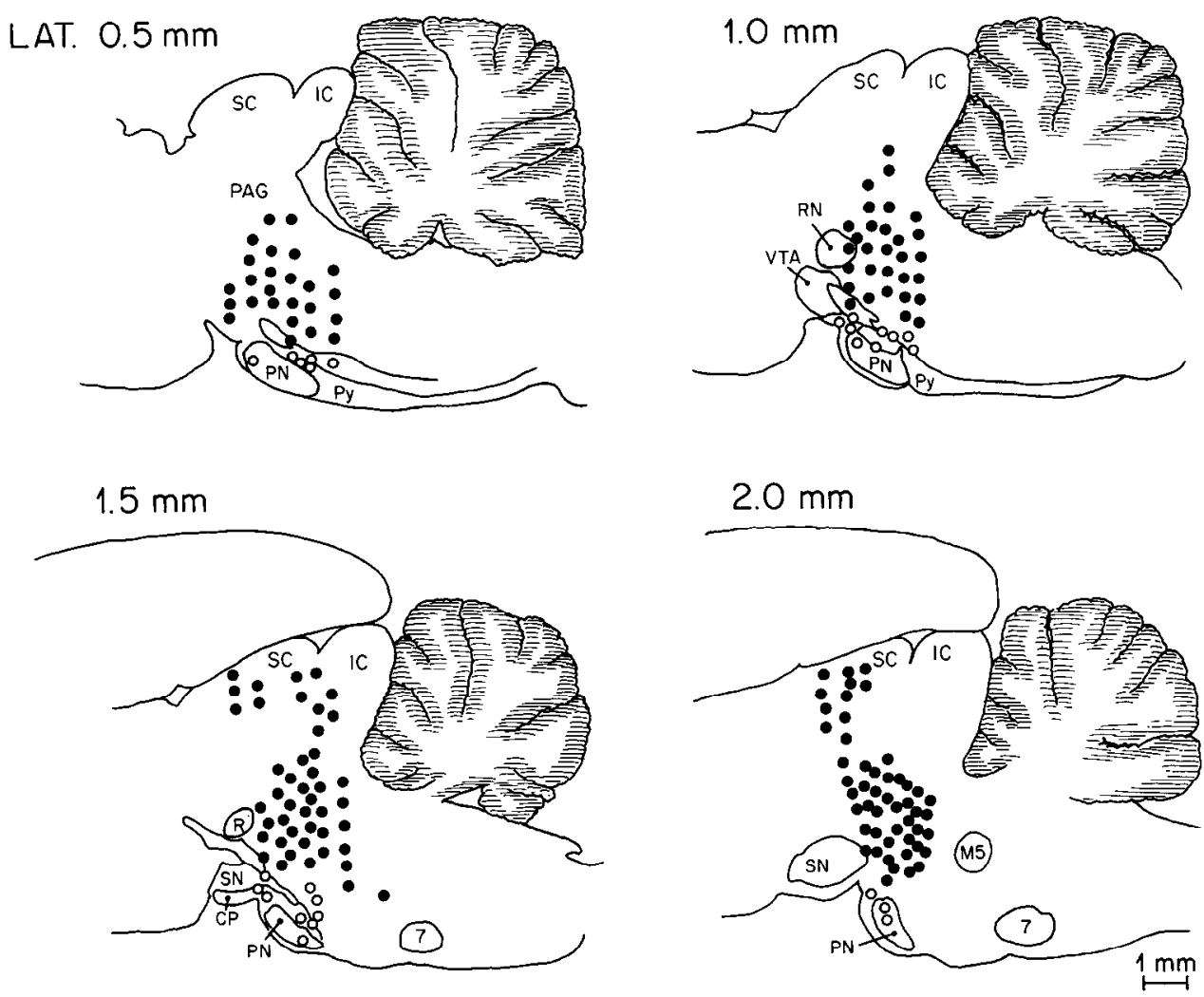

Figure 1. Location of brain-stem loci that produced complete suppression of RDA at low-intensity stimulation. Sagittal sections of brain stem are shown at $0.5,1.0,1.5,2.0 \mathrm{~mm}$ from midline. Pooled data from 4 representative animals. Filled circles represent sites that produced complete suppression of $\mathrm{RDA}$ at intensities less than $50 \mu \mathrm{A}$ within 1 sec of onset of the brain-stem stimulus; open circles represent sites that evoked RDA activity. Although widespread areas of brain stem produced suppression of RDA, the predominate locations of these suppressive sites were in the rostral pontine and mesencephalic reticular formation. activated, produced a permanent phase resetting of the ongoing RDA rhythm and, therefore, whether they are capable of controlling the neuronal networks responsible for cycle timing (Lennard, 1985).

The EMG was full-wave-rectified and filtered $(80 \mathrm{~Hz}$ low-frequency cutoff). For each experiment the data from 2-4 of the lowest threshold sites for suppression of RDA were recorded on an 8 channel Vetter FM tape recorder for subsequent quantitative analysis. Threshold for complete RDA suppression within $1 \mathrm{sec}$ of brain-stem stimulus onset for each loci within a given electrode tract was determined by initially adjusting the brain-stem stimulus to $50 \mu \mathrm{A}$. If complete suppression occurred, then the stimulus intensity was reduced to $25 \mu \mathrm{A}$ and a new trial initiated. Subsequent trials were repeated with intensity increments of 5-10 $\mu \mathrm{A}$ until the threshold for complete suppression of RDA within $1 \mathrm{sec}$ of the onset of the brain-stem stimulus was obtained. Intertrial intervals were $30 \mathrm{sec}$ to $1 \mathrm{~min}$. After the threshold was determined the electrode was lowered $0.5 \mathrm{~mm}$ and the threshold for that site determined. For a determination of the effects of continuous brain-stem stimulation on cycle characteristics, data analysis consisted of cycle-by-cycle measurements of CD, BD, and I-EMGs for at least 15 consecutive RDA cycles by an IBM-XT computer with software developed by RC Electronics (Santa Barbara, Calif.). Data were sampled at $1 \mathrm{KHz}$. Means and SD were calculated. To determine the effects of short pulse trains on the RDA cycle characteristics at least 10 consecutive stimulus perturbations at a given phase of the cycle were measured and means and SD were calculated. Each perturbed cycle was separated by 4-6 nonperturbed cycles. Statistical analyses consisted of block design analyses of variance tests with post hoc tests for determination of significant differences between means utilizing the method of Newman-Keuls (Kirk, 1982). In all cases the level of significance was chosen at $p \leq 0.05$.

Upon completion of each experiment the animal was sacrificed with an overdose of urethane and the brain perfused with saline followed by $10 \%$ formalin. The brain was fixed subsequently in $10 \%$ formalin for a week. Frozen celloidin sections were cut at $80 \mu \mathrm{m}$ sections and stained for Nissl substance with carbol fuchsin for subsequent verification of electrode positions.

\section{Results}

The distribution of low-threshold suppressive brain-stem sites on RDA is shown in the sagittal sections of Figure 1. The pre- dominate effects of continuous low-intensity trains at $50 \mathrm{~Hz}$ to brain-stem loci within the rostral pons and midbrain were to suppress ongoing cortically induced RDA and the accompanying RJMs. Complete suppression of ongoing RDA within 1 sec of the onset of brain-stem stimulation was produced at lowintensity stimulation ( $<50 \mu \mathrm{A}$, filled circles) by sites ventral and lateral to the periaqueductal gray of the midbrain. The lowest threshold sites were consistently found to be localized to the reticular formation of the midbrain and continuous with reticular nuclei of the pons. Adjacent sites produced similar effects but at higher stimulus intensities and were generally accompanied by gross body movements. Stimulation of any of these suppressive sites at stimulus intensities less than $50 \mu \mathrm{A}$, in the absence of cortically induced RDA, did not produce any movement of the animal or EMG activity in jaw closer muscles. Visual inspection of the mandible in the absence of cortical stimulation showed no change in position during the brain-stem stimulus. In some animals stimulation of sites within the lateral borders of the superior colliculus suppressed ongoing RDA. In some trials, brain-stem stimulation produced rhythmical activity in the hindlimbs resembling locomotion. These sites were usually located in the midbrain $1.5-2.5 \mathrm{~mm}$ lateral to the midline and 3-5 mm deep from the brain-stem surface. Interestingly, no sites produced facilitation (increase in amplitude of the EMG or decrease in CD) of the ongoing RDA at the intensities studied. In fact, the only sites that produced activation of RDA were in the vicinity of the cerebral peduncle (Fig. 1, open circles). Presumably this effect was due to direct activation of corticobulbar fibers (Nozaki et al., 1986).

The effects of varying stimulus current intensity to a representative suppressive brain-stem site on the EMG and RDA cycle parameters are shown in Figures 2 and 3. Figure 2 shows the effects of varying current intensity on the EMG activity. In 

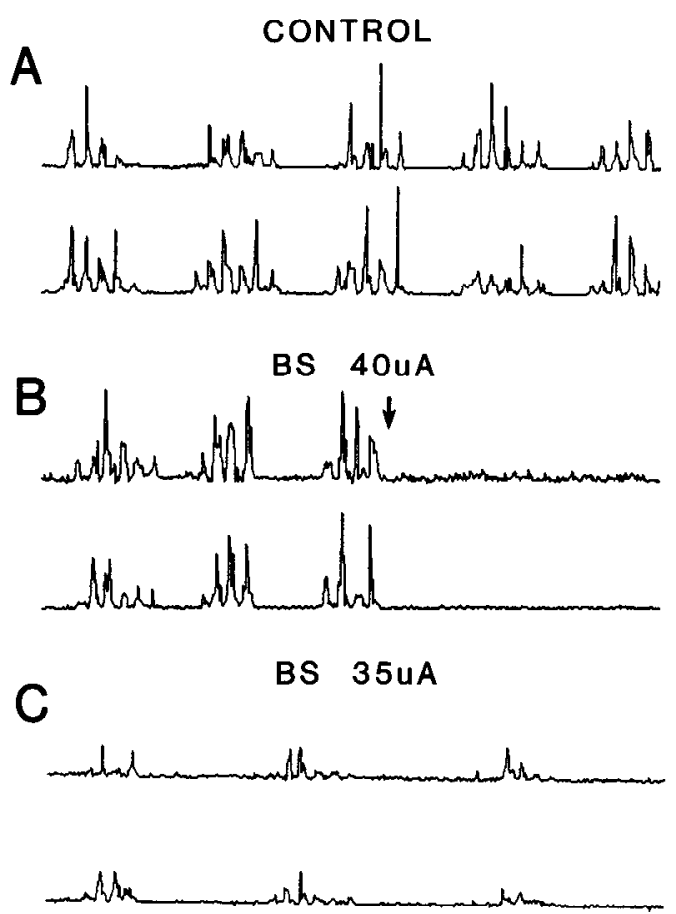

D
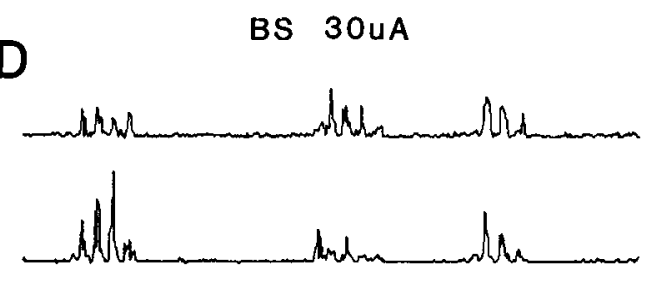

Figure 2. Effects of varying stimulus intensity to a representative suppressive brain-stem site on cortically evoked RDA. $A$, Control RDA evoked by cortical stimulation. $B$, Stimulation of brain stem during cortically evoked RDA. Arrow indicates onset of repetitive stimulation of the brain stem at $40 \mu \mathrm{A}$. $C$, Effects of $35 \mu \mathrm{A}$ brain-stem stimulation on RDA. Trace taken approximately $1 \mathrm{sec}$ after onset of brain-stem stimulus. $D$, Effects of $30 \mu \mathrm{A}$ brain-stem stimulation on RDA. Trace taken approximately $1 \mathrm{sec}$ after onset of brain-stem stimulus. For $B$ $D$, brain-stem stimulation parameters were repetitive pulses of $50 \mathrm{~Hz}$ with a pulse duration of $0.15 \mathrm{msec}$. Time calibration $(D), 100 \mathrm{msec}$.

this example, the threshold for complete RDA suppression was $40 \mu \mathrm{A}$ (Fig. $2 B$ ). The most consistent and significant changes observed (10 animals) were an increase in CD and a decrease in EMG amplitude (Fig. $2 C, D$ ) prior to complete suppression (Fig. 2B). Figure 3 shows a graphic analysis of the data presented in Figure 2. In Figure $3 A$ the relationship between $C D$ and stimulus current intensity is shown. An increase in $C D$ was obtained with increasing current intensities. Changes in EMG amplitude were determined by plotting the ratio of integrated EMG to burst duration (I-EMG/BD) as a function of current intensity (Fig. 3C). A decrease in I-EMG/BD was observed prior to complete RDA suppression. The relationship between BD and stimulus current is shown in Figure $3 B$. Although the duration of individual DIG EMG bursts during the RDA cycle was generally shorter compared to controls, these changes were usually small compared to the changes seen in CD or I-EMG/ BD. Interestingly, brain-stem activation also produced an increase in variability of the rhythmical EMG output, as evidenced by an increase in variability of $\mathrm{CD}$ and $\mathrm{BD}$ with increasing stimulus intensity. These relationships between cycle parameters and current intensity were observed in all animals tested $(n=5)$.

We have previously argued that cortically induced RJMs are produced by the simultaneous activation of a series of polysynaptic corticotrigeminal pathways and a central pattern generating network that directly modulates the excitability of these corticotrigeminal pathways (Chandler and Goldberg, 1982; Chandler et al., 1985a). To determine if activation of these same suppressive brain-stem sites produced a decrease in excitability of the short-latency polysynaptic corticotrigeminal pathways to DIG motoneurons, the effects of repetitive stimulation of these suppressive brain-stem sites on the short-latency DIG response induced by SPT stimulation of the cortex were examined. Figure $4 B$ shows the effects on the averaged EMG evoked by SPT cortical stimulation of a continuous $50 \mathrm{~Hz}$ stimulation to a representative suppressive brain-stem site that produced suppression of RDA activity at less than $50 \mu \mathrm{A}$. The effects of varying the intensity of the continuous brain-stem stimulation on the SPT evoked averaged peak amplitude EMG are shown in Figure $4 A$. In this example the averaged peak amplitude is expressed as a percentage of the control value. In all animals examined ( $n=10)$, a decrease in the averaged amplitude of the short-latency DIG EMG was observed from stimulation of any of the sites that produced a suppression of the RDA at less than $50 \mu \mathrm{A}$.

In order to determine if these suppressive brain-stem sites also produced direct effects on the neuronal networks responsible for RDA oscillation, stimulus-perturbation experiments were performed in an additional 5 animals. Trains $(50-100 \mathrm{~Hz})$ of $50-100 \mathrm{msec}$ stimuli were delivered to the brain stem at different phases of the RDA cycle, and their effects on the duration of the perturbed cycle ( $P$ cycle) and the subsequent cycles $(\mathrm{P}+1, \mathrm{P}+2$, etc.) were compared to the preceding control cycles $(\mathrm{P}-1$ cycle). In Figure $5 A$ the $\mathrm{P}-1, \mathrm{P}$, and $\mathrm{P}+1$ cycles are defined in relation to the onset of the stimulus perturbation (upper trace). For each phase, at least 10 perturbing stimuli were given during a given RDA trial. A trial consisted of at least 15 sec of continuous cortical stimulation at $40 \mathrm{~Hz}$. In some cases the data from 2 consecutive trials were pooled. Figure 5, $A$ and $B$, illustrates the effects on the EMG cycle characteristics of a 90 msec stimulus train delivered at 2 different phases of the RDA cycle from a representative animal. Regardless of the phase of the cycle in which the stimulus occurred, the onset of the subsequent DIG EMG burst was delayed as compared to the preceding control cycle. This was consistently observed in all 5 animals tested. In Figure $6 A$ the relationship between $C D$ and cycle phase in which the stimulus occurred is plotted. The durations of the mean $P$ cycle and the $P+1$ cycle are expressed as a percentage of the mean preceding $P-1$ cycle (control). A value of 100 indicates that no change in the duration of the cycle occurred. A value less than 100 indicates that the $C D$ was less than the control, while a value greater than 100 indicates that the CD was longer than control. When the stimulus was applied early in the RDA cycle, during an ongoing DIG EMG burst, the burst was reduced in amplitude and duration (Fig. $5 A$ ). Interestingly, when the stimulus was applied at these early phases the subsequent burst onset did not come earlier than expected. In fact, it was generally the case that the burst following the perturbing stimulus train came later than expected, thus increasing the duration of the overall $\mathrm{CD}$ of the perturbed cycle 
A

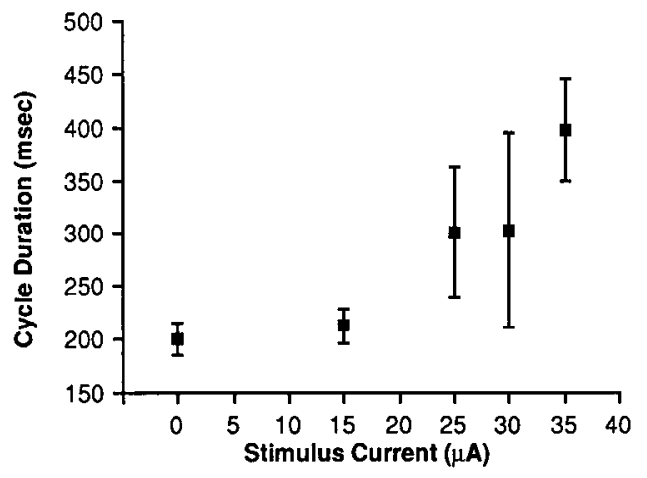

C

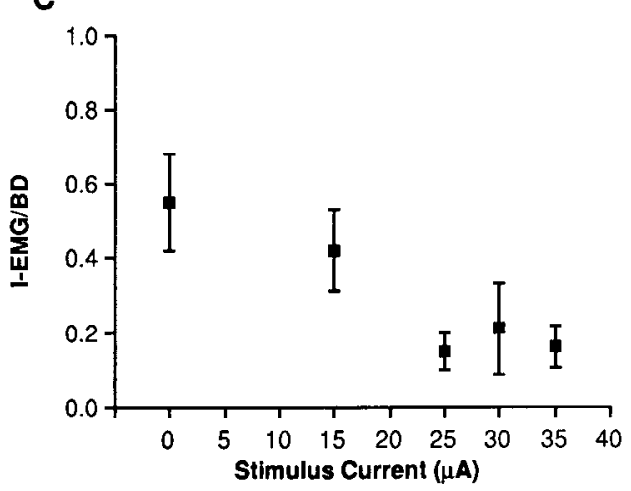

B

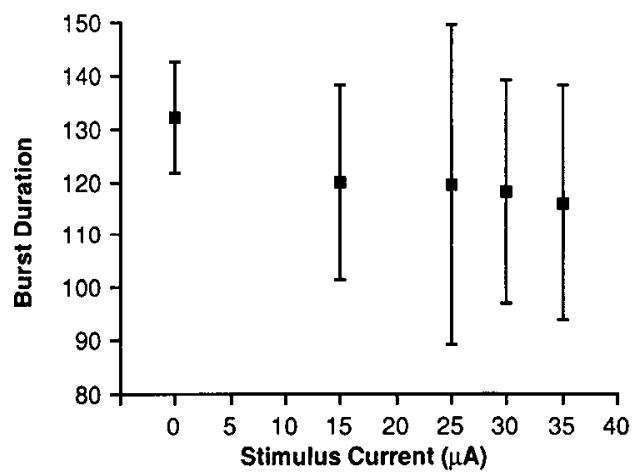

Figure 3. Effects of varying brain-stem stimulus intensity on RDA cycle characteristics. $A$, Plot of mean RDA cycle duration $(C D)$ versus brain-stem stimulus current intensity. $B$, Plot of DIG BD versus stimulus current intensity. $C$, Plot of normalized integrated EMG (I-EMG/BD) versus stimulus intensity. Data taken from same animal as in Figure 2. Each point represents the mean and SD of at least 15 consecutive cycles. In this example, a $40 \mu \mathrm{A}$ stimulus to the brain stem produced complete suppression of RDA.
(Fig. 6A, dark squares). When the perturbing stimulus was delivered at progressively later phases of the cycle, the subsequent onset of the next cycle was significantly delayed. A maximum delay was usually obtained when the stimuli were given at phases between 0.7 and 1.0 of the cycle. These results were consistently observed in all animals tested $(n=5)$.

In order to determine if a phase resetting of the oscillator occurred as a result of the increase in duration of the $\mathbf{P}$ cycle as opposed to changes in excitability of the motoneurons or output neurons of the central oscillator network, the durations of the subsequent $P+1$ and $P+2$ cycles were examined. A compensatory decrease in duration of those cycles would suggest that a phase resetting had not occurred (Lennard, 1985). Figure $6 A$ (open squares) shows graphically the effects of the perturbing stimulus on the subsequent $P+1$ cycle duration as expressed as a percentage change from control. As observed, compared to the control cycle, the $\mathrm{P}+1$ cycle duration was not changed significantly regardless of the phase of the cycle the stimulus was administered. This is shown more clearly in Figure 6, B and $C$, where the mean $C D$ is plotted as a function of cycle number for a given phase of the cycle the stimulus was applied. In Figure $6 B$ the phase the stimulus was applied was 0.1 while in Figure $6 C$ it was 0.7. Regardless of the phase the stimulus was applied, the stimulus significantly increased the duration of the perturbed cycle ( $\mathrm{P}$ cycle) but produced no statistical change in duration of the subsequent $\mathbf{P}+1$ or $\mathbf{P}+2$ cycles, as compared to the control $(P-1)$ cycles. These effects were observed in all animals tested $(n=5)$. These data indicate that the perturbing stimulus produccd a permanent resetting of the RDA cycle (Lennard, 1985).

The conclusion that brief activation of suppressive brain-stem sites produced a resetting of the RDA rhythm is further sup-
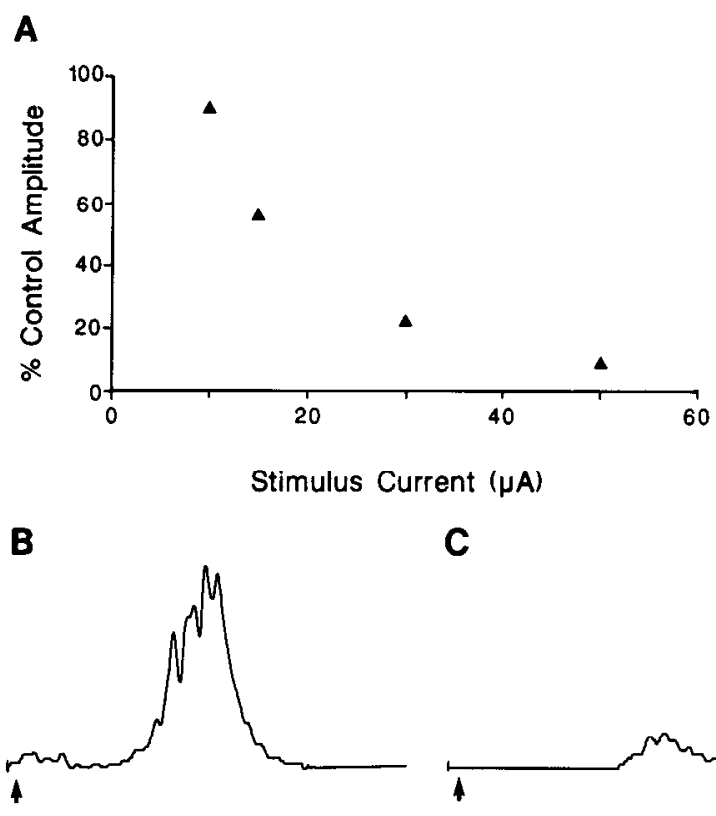

C

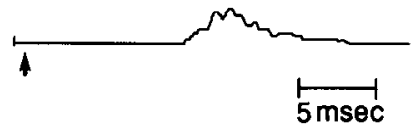

Figure 4. Effect of varying stimulus intensity to the brain stem on mean amplitude of short-latency DIG response evoked by short pulse train stimulation of the masticatory cortex. Cortical stimulus train: 3 pulses, $500 \mathrm{~Hz}, 0.3 \mathrm{msec}$. $A$, Plot of mean DIG amplitude $(n=16)$ versus brain-stem stimulus intensity. Amplitude of DIG is expressed as a percentage of control amplitude. A value of 100 represents no change from control. $B$, Example of $50 \mu \mathrm{A}$ repetitive brain-stem stimulus on DIG EMG response evoked by short pulse train stimulus to cortex. Arrow indicates onset of cortical stimulus train. Stimulus parameters same as in $A$. Data taken from a brain-stem sitc that produced suppression of RDA at less than $50 \mu \mathrm{A}$. 
Figure 5. Effect of short pulse train brain-stem stimulus perturbation at different phases of cycle on subsequent RDA. $A$, Stimulus perturbation early in RDA cycle. The cycle in which the stimulus perturbation occurred is termed the $\mathbf{P}$ cycle, while the cycle preceding the stimulus perturbation is termed the $\mathbf{P}-1$ cycle and served as the control cycle. Cycles that occurred after the $\mathbf{P}$ cycle are termed the $\mathbf{P}+1$, $\mathrm{P}+2$, cycles, etc. Upper trace in $A, B$, and the following figures represent occurrence of brain-stem stimulus train. $B$, Same as $A$ except stimulus perturbation occurred late in RDA cycle. Note that for both $A$ and $B$, regardless of whether the stimulus occurred early or late in cycle, the subsequent onset of the next DIG burst was delayed compared to the preceding control cycle. Brain-stem stimulus train: 9 pulses, 100 $\mathrm{Hz}, 0.15 \mathrm{msec}, 50 \mu \mathrm{A}$.
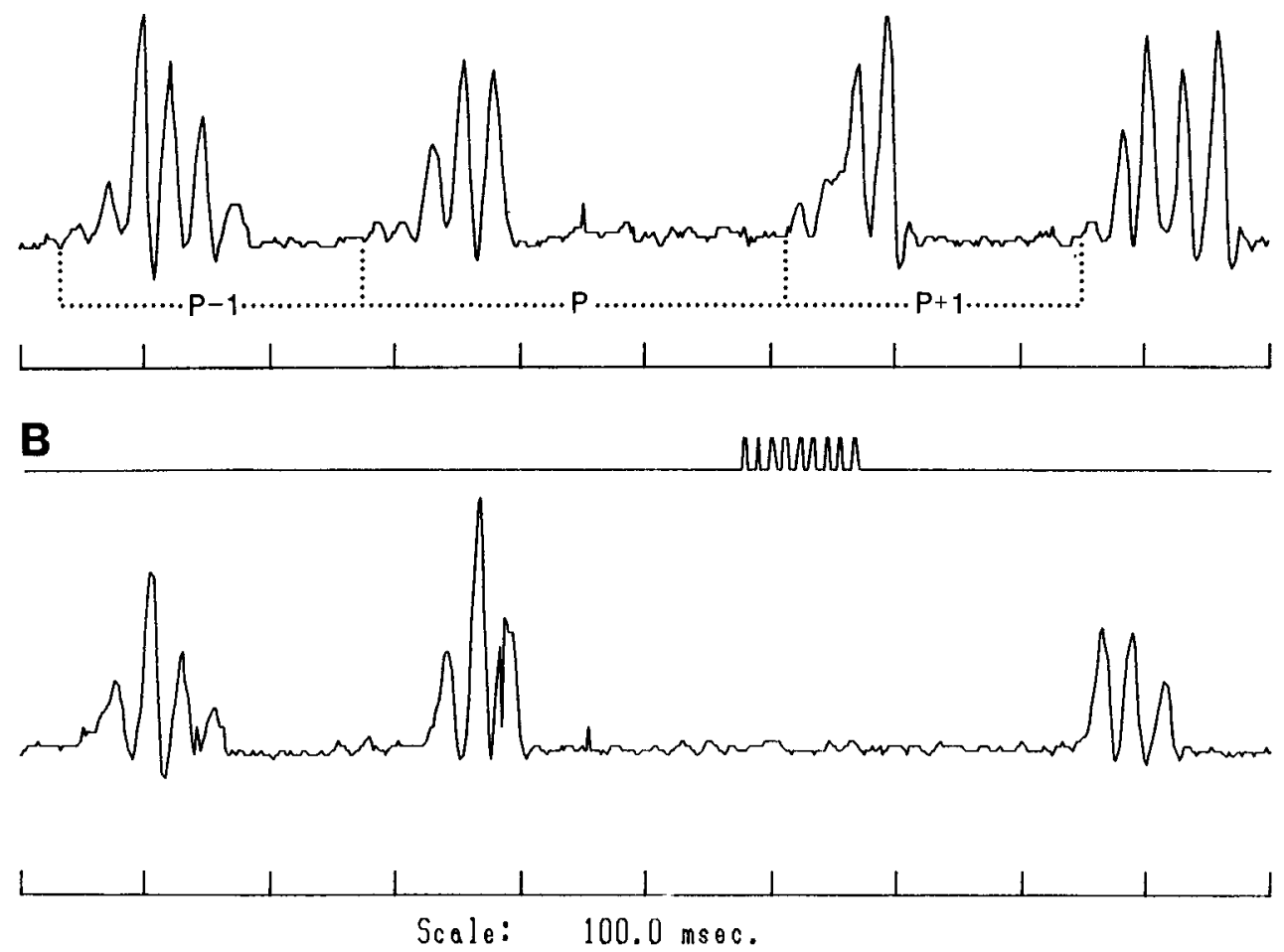

ported by examining the effects on $C D$ of varying stimulus train duration, while keeping the phase of stimulus delivery constant. Figure 7 presents data from another animal showing the effects on rhythmical DIG EMG activity of varying pulse durations. The phase of cycle the stimulus was applied was approximately 0.5 and occurred after the termination of the preceding DIG EMG burst. A graphic analysis of the data of Figure 7 is shown in Figure 8. A progressive increase in the stimulus train duration produced a significant increase in the mean duration of the ongoing perturbed cycle (Fig. 8A, $\mathrm{P}$ cycle, filled circles) while producing no statistical change, as compared to control cycles ( $P-1$, open squares), in the subsequent mean $P+1$ cycle duration (filled squares). Figure $8 B$ shows the effects of varying train duration on the subsequent DIG EMG BD. No statistical difference in the subsequent $\mathrm{BD}$ from the preceding control $\mathrm{BD}$ was found regardless of the duration of the stimulus train.

\section{Discussion}

The major findings of this study were that widespread areas of the rostral pons and midbrain can suppress activity in both the short-latency jaw opening pathways originating in masticatory cortex and on the neuronal networks responsible for oscillation and muscle timing during RJM activity induced by repetitive cortical stimulation. Although with the electrical stimulation techniques used in the present study we cannot, and have not made any attempt to, precisely attribute these findings to particular brain-stem loci, the most consistent sites with the lowest thresholds for RJM inhibition were in the regions of the rostral pontine and mesencephalic reticular formation (Fig. 1). There is only 1 other report describing the effects of stimulation of brain-stem loci on RJM activity in the anesthetized preparation (Sumi, 1971). In that study it was also found that widespread areas of pons and midbrain produced suppression of cortically induced RJMs. In the present study, facilitatory responses (decreases in CD, increases in EMG amplitude) were not observed. This could be due to the low-intensity brain-stem stimulation used, which might be selectively activating a particular class of neuron. It was observed that at intensities greater than $50 \mu \mathrm{A}$ whole-body movements or escapelike responses were elicited quite often. These responses could have masked a facilitatory effect on the circuits controlling RJMs.

In previous studies we argued that a central pattern-generating (CPG) network(s), activated by masticatory cortex, phasically modulates the excitability of DIG premotoneurons, which themselves are activated directly by masticatory cortex, to produce rhythmical jaw opening during repetitive cortical stimulation (Chandler and Goldberg, 1982; Chandler et al., 1985a; Lambert et al., 1985). Furthermore, it was suggested that these premotoneurons are not a component of the CPG network(s) but merely output neurons of that network(s) (Chandler and Goldberg, 1982; Chandler et al., 1985a). The mechanism underlying the brain-stem suppression of the short-latency jaw opening response and RDA induced by cortical stimulation observed in the present study could be due to (1) pre- or postsynaptic inhibition of DIG motoneurons or premotoneurons, or (2) inhibition of ncurons comprising the brain-stem CPG nctwork(s), or (3) some combination of both.

The present data indicate that these rostal pontine and midbrain sites produce a decrease in excitation of neurons comprising both the short-latency disynaptic corticotrigeminal pathways and the CPG network(s) responsible for oscillation. The excitability of the short-latency jaw opening pathways, when activated in the absence of RJMs, was reduced by repetitive activation of these brain-stem sites (Fig. 4). This observation 

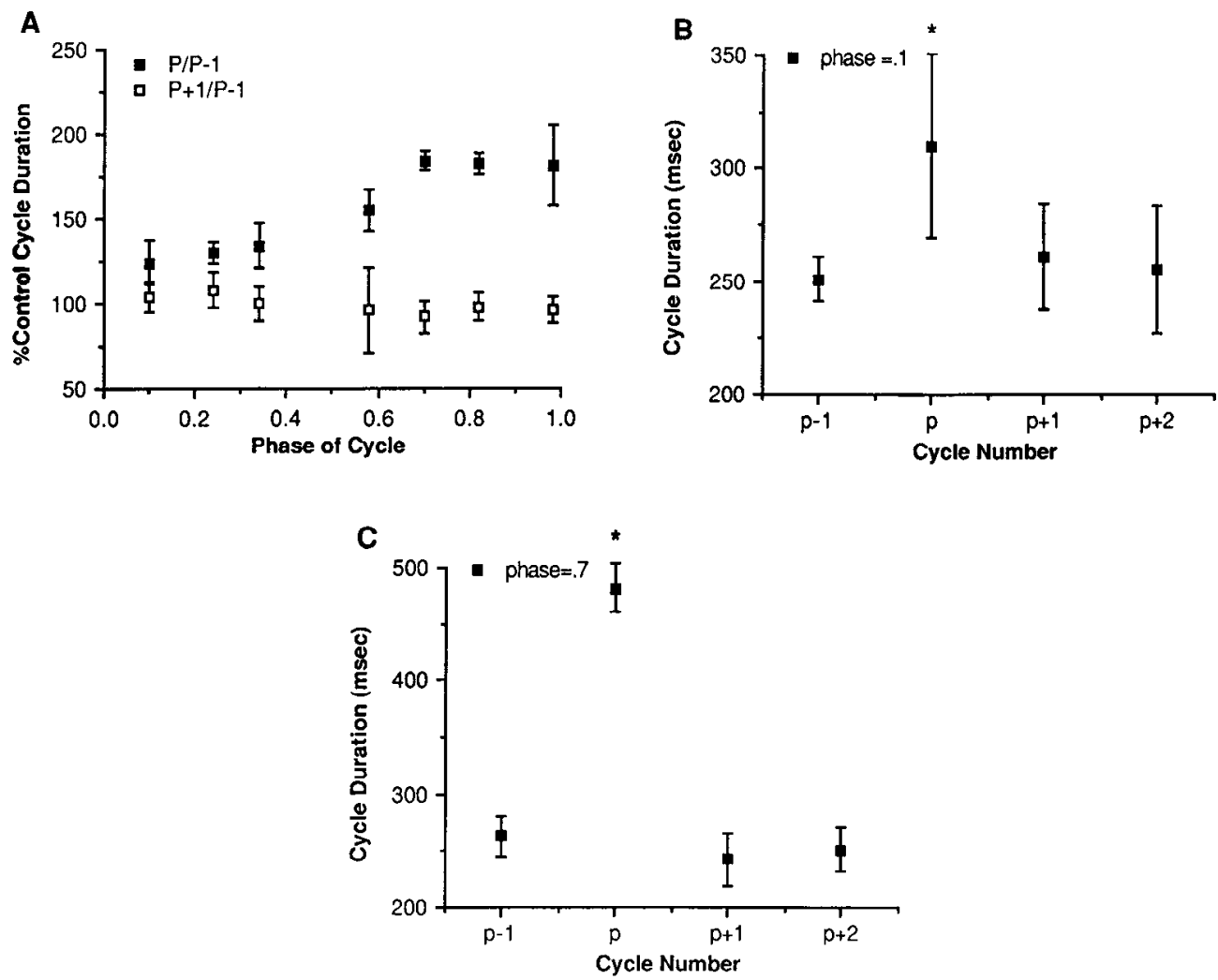

Figure 6. Effect of varying the phase of the cycle the stimulus perturbation occurred on mean RDA cycle characteristics. Data taken from same animal shown in Figure 5. $A$, Plot of mean $\mathrm{CD}$ of $\mathrm{P}$ cycle and $\mathrm{P}+1$ cycle versus phase of cycle the brain-stem perturbation occurred. $\mathrm{P}$ and $\mathrm{P}+1$ CDs are plotted as a percentage of control cycle $(P-1)$. A value of 100 represents no change from the control $C D$ while a value greater than 100 represents a prolongation of the cycle. A 2-way ANOVA (phase $\times$ cycle number) and subsequent post hoc analysis showed that mean CD of the $\mathrm{P}$ cycle was significantly affected by varying the phase in which the stimulus perturbation occurred $\left(F_{6,59}=12.3, p<0.001\right)$. Post hoc analysis showed no difference between the mean $P+1 C D$ and control $(P-1$ cycle) regardless of phase the stimuli were applied. Since data are expressed as a percentage of control, the error bars in this example represent the coefficient of variation. $B$, Plot of mean $C D$ versus cycle number for a stimulus perturbation evoked at the 0.1 phase of the RDA cycle. $C$, Same as $B$, except the stimulus perturbation occurred at the 0.7 phase of the RDA cycle. For both $B$ and $C$, post hoc analyses showed that the stimulus significantly $(p<0.01)$ increased the mean duration of the $P$ cycle as compared to the control $P-1 \mathrm{CD}$. The mean duration of the subsequent $P+1$ and $P+2$ cycles did not differ from control. Each point represents the mean and SD of at least 10 cycles.

could, in part, explain the observed suppression of RJMs by repetitive stimulation of these sites (Fig. 2) but would not account for the phase-resetting of the cycle that was observed during short pulse train stimulation during various phases of the RDA cycle.

The phase-resetting experiments in the present study suggest that, in addition to suppression of activity in neurons comprising the polysynaptic corticotrigeminal pathways from rostral brain-stem sites, a component of the RJM suppression must also involve inhibition of neurons within the CPG network(s) itself. Short pulse train stimulation of these sites during any phase of the RJM cycle was found to produce a delay in onset of the next DIG EMG burst without a compensatory shortening in the $P+1$ or $P+2 C D$ (Fig. 6). This was observed in all 5 animals tested. These experiments suggest that rostral brainstem nuclei can control both the amplitude of DIG EMG activity and the parameters of cycle oscillation. In earlier studies on the brain-stem mechanisms controlling locomotion it was suggested that the amplitude of EMG activity, as opposed to cycle timing parameters, was controlled by activity in brain-stem loci (Orlovsky, 1972). Recent studies, however, showed that pontine and medullary reticular formation sites can control the amplitude and the duration of the step cycle in thalamic cats (Drew and Rossignol, 1984) during controlled locomotion induced by midbrain stimulation or during fictive locomotion in decorticate preparations (Russell and Zajac, 1979).

There are inherent problems, of course, in the interpretation of studies utilizing electrical stimulation of nonhomogeneous structures. Current spread to adjacent structures and stimulation of ascending and descending fibers of passage or their collaterals certainly pose problems to the precise determination of the origin of the site(s) responsible for the effect. In the present study, the effects of current spread were minimized by the use of lowintensity stimulation $(\leq 50 \mu \mathrm{A})$ through microelectrodes. With the type of stimulating electrode used in the present study, this type of stimulation, conservatively, should produce effective current spread of less than $500 \mu \mathrm{m}$ (Ranck, 1975; Bagshaw and Evans, 1976). It is unlikely that these effects are mediated solely through ascending cortical and subcortical pathways since we observed these effects in prccollicular, decerebrate preparations during RJMs evoked by corticobulbar fiber stimulation (unpublished observations). Furthermore, the fact that widespread areas were shown to produce suppression at relatively low threshold intensities suggests that these effects are mediated by multiple brain-stem loci as opposed to current spread to a single suppressive site.

The precise pathways mediating the suppression from rostral pontine and midbrain sites are not known. As mentioned pre- 
A

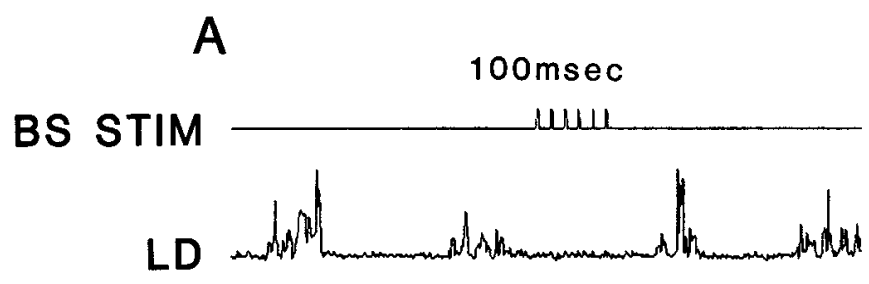

B

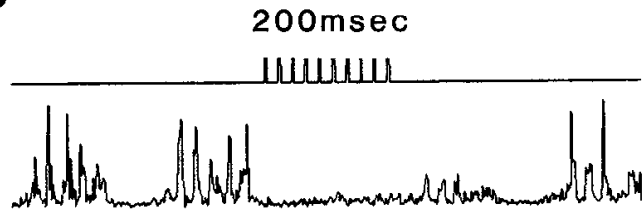

C

$250 \mathrm{msec}$

ишишин

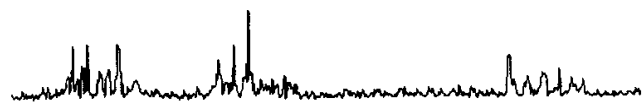

D
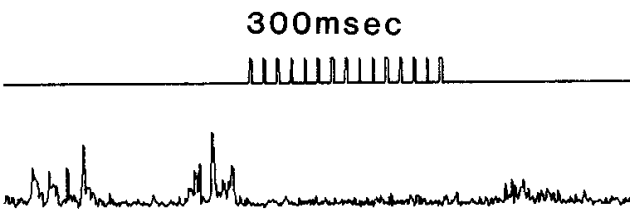

Figure 7. Effects of varying brain-stem stimulus pulse duration on RDA. A, A $100 \mathrm{msec}$ duration stimulus train delivered to the brain stem. Stimulus occurred at approximately the termination of the previous DIG burst. $B-D$, Same as $A$, except stimulus pulse durations were 200,250 , and $300 \mathrm{msec}$, respectively. For traces $A-D$, brain stem stimulus was $50 \mathrm{~Hz}, 0.15 \mathrm{msec}$ per pulse. Note progressive delay of occurrence and decrease in EMG amplitude of subsequent DIG burst as pulse duration increased. Time calibration in $D, 90 \mathrm{msec}$.

viously, Sumi (1971) found that cortical RJMs were suppressed by deep layers of the superior colliculus, periaqueductal gray (PAG), and surrounding reticular formation. In those experiments, the suppression was sometimes accompanied by an in- crease in tonic jaw closer muscle activity. It was proposed that the suppression of RJMs by brain-stem sites was due to either activation of a nonspecific inhibitory reticular formation pathways or to an indirect reflex activation of inhibitory interneurons as a result of an increase in jaw closer muscle activity. In the present study, this latter hypothesis is unlikely. Although jaw movements were not monitored, visual inspection of the position of the mandible showed that the suppression of RJMs was accompanied by a return to rest position of the mandible. In addition, incrcascd activity in jaw closer muscles was never observed at the stimulus intensities used.

The midbrain PAG and surrounding reticular formation have been implicated as systems involved with pain suppression (reviewed in Basbaum and Fields, 1978). The PAG of the midbrain upon stimulation was shown to inhibit the jaw opening reflex induced by intraoral stimulation (Oliveras et al., 1974; Sessle and $\mathrm{Hu}, 1981$; Sessle et al., $1981 \mathrm{a}, \mathrm{b}$; Dostrovsky et al., 1982; Hayashi et al., 1984). It was shown, subsequently, that adjacent regions of midbrain reticular formation were capable of producing suppression of the jaw opening reflex (Dostrovsky et al., 1982). There is evidence to suggest that these suppressive effects are mediated by a relay in either the medullary reticular formation or the medullary nucleus raphe magnus (Basbaum and Fields, 1978; Dubner et al., 1978; Gebhart et al., 1983) and directed towards trigeminal sensory nucleus second-order interneurons (Sumino, 1971), which mediate the jaw opening reflex. Postsynaptic inhibition of DIG motoneurons also cannot be ruled out. It is not likely that the results of this study represent the effects of trigeminal sensory nuclei inhibition since these nuclei are most likely not involved with the genesis of cortical RJM activity (Dubner et al., 1978; Luschei and Goldberg, 1981; Chandler and Tal, 1986). In the present study the suppression of the short-latency jaw opening response induced by short pulse train cortical stimulation is most likely due to postsynaptic inhibition of either DIG motoneurons or premotoneurons, although a mechanism of presynaptic inhibition cannot be excluded. It is unlikely that monosynaptic inhibition from midbrain sites is responsible for these effects since there are few direct projections to the trigeminal motor nucleus from these regions (Mizuno et al., 1983; Travers and Norgren, 1983; Vornov and Sutin, 1983). Neurons have been identified within the rostral medullary and caudal pontine reticular formation which were orthodromically activated by cortex and antidromically activated by trigeminal motor nucleus stimulation (Nozaki et al.,
Figure 8. Effects of varying brain-stem stimulus pulse duration on RDA cycle characteristics. Data from animal shown in Figure 7. $A$, Plot of mean $C D$ versus stimulus pulse duration for $\mathrm{P}-1, \mathrm{P}$, and $\mathbf{P}+1$ cycles. A 2-way ANOVA (pulse duration $\times$ cycle number) showed that pulse duration had significant affects on the mean $\mathrm{CD}\left(F_{4,64}=40.2\right.$, $p<0.001)$. Post hoc analyses showed that the mean $C D$ for the $P$ cycle was significantly greater than the $P-1$ and $P+1$ CDs. $B$, Plot of duration of DIG burst occurring in $P+1$ cycle versus stimulus pulse duration. Varying pulsc duration did not significantly change mean BD of DIG burst occurring in $\mathrm{P}+1$ cycle as compared to the control $P-1$ burst.
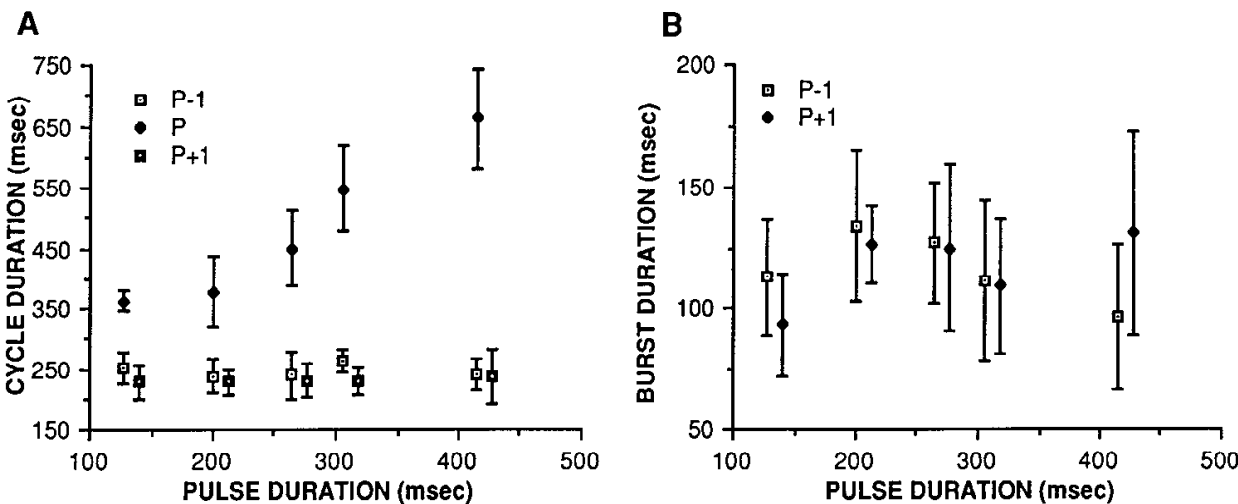
1983). It was proposed that those cells mediate the short-latency jaw opening response from stimulation of masticatory cortex (Nakamura et al., 1980; Nozaki et al., 1983). Furthermore, stimulation of this area produces excitatory and inhibitory synaptic potentials in DIG motoneurons (Nakamura et al., 1975, 1979; Takatori et al., 1981; Nozaki et al., 1983). It is possible that the suppression of RDA by rostral brain-stem sites observed in the present study is mediated by activation of a population of inhibitory premotoneurons within the lower pontine RF that were previously identified by Nakamura et al. (1975) to produce a long-latency hyperpolarization in DIG motoneurons or to direct postsynaptic inhibition of the excitatory DIG premotoneurons within this region. Many of these midbrain sites have direct projections to pontine and medullary reticular formation sites (Edwards, 1975), precisely the regions where these trigeminal premotoneurons were identified both anatomically (Mizuno et al., 1983; Travers and Norgren, 1983; Vornov and Sutin, 1983) and electrophysiologically (Nozaki et al., 1983) and where the central networks responsible for jaw oscillation, presumably, are located (Chandler and Tal, 1986; Nozaki et al., 1986). It is also possible that these inhibitory reticular formation neurons receive convergent inputs from pain-modulating systems originating in the midbrain and contribute to the suppression of the jaw opening reflex induced by intraoral stimulation.

Previous studies have implicated the mesencephalic reticular formation and $P A G$ as being part of an integral system involved with suppression of nociceptive input (Basbaum and Fields, 1978, for review). Recently, it was shown that widespread areas of the brain stem can suppress nonnociceptive trigeminal reflex pathways as well as nociceptive reflex pathways (Oliveras et al., 1974; Sessle and Hu, 1981; Sessle et al., 1981a, b; Dostrovsky et al., 1982; Hayashi et al., 1984), suggesting a lack of functional specificity for these sites. The present data demonstrate that these loci can influence other complex oral movement behaviors that do not necessarily involve nociceptive systems.

Although the midbrain reticular formation and PAG are not part of the neuronal circuitry responsible for RJMs in the guinea pig (Chandler and Goldberg, 1984; Chandler and Tal, 1986; Nozaki et al., 1986), the present data demonstrate that these sites are capable of modulating, potently, the circuits responsible for controlling frequency and timing as well as amplitude of DIG EMG activity during cortically induced RJMs in the guinea pig. It is not surprising that these areas can influence jaw movements when one considers that the expression of RJMs evoked by systemic dopaminergic activation in the anesthetized (Chandler and Goldberg, 1984; Chandler et al., 1985b) or unanesthetized preparation (Redgrave et al., 1980) depends upon the integrity of the superior colliculus and/or surrounding reticular formation, sites that are targets of substantia nigra outflow (Beckstead et al., 1979). It is possible that the control and modulation of the central timing circuitry could arise in basal ganglia and associated structures since superior colliculus and surrounding reticular formation have been implicated as output stations of basal ganglia circuitry via a synapse in substantia nigra ( $\mathrm{Di}$ Chiara et al., 1978).

\section{References}

Bagshaw, E. V., and M. H. Evans (1976) Measurement of current spread from microelectrodes when stimulating within the nervous system. Exp. Brain Res. 25: 391-400.

Basbaum, A., and H. L. Fields (1978) Endogenous pain control mechanisms: Review and hypothesis. Ann. Neurol. 4: 451-462.
Beckstead, R. M., V. B. Domesick, and W. J. H. Nauta (1979) Efferent connections of the substantia nigra and ventral tegmental area in the rat. Brain Res. 175: 191-217.

Chandler, S. H., and L. J. Goldberg (1982) Intracellular analysis of synaptic mechanisms controlling spontaneous and cortically induced rhythmical jaw movements in the guinea pig. J. Neurophysiol. 48: 126-138.

Chandler, S. H., and L. J. Goldberg (1984) Differentiation of neural pathways mediating cortically induced dopaminergic activation of the central pattern generator (CPG) for rhythmical jaw movements in the anesthetized guinea pig. Brain Res. 323: 297-301.

Chandler, S. H., and L. J. Goldberg (1986) Effects of brainstem activation on cortically induced rhythmical jaw movements in the guinea pig. Soc. Neurosci. Abstr. 12: 884 .

Chandler, S. H., and M. Tal (1986) The effects of brain stem transections on the neuronal networks responsible for rhythmical jaw muscle activity in the guinea pig. J. Neurosci. 6: 1831-1842.

Chandler, S. H., L. J. Goldberg, and B. Alba (1985a) Effects of a serotonin agonist and antagonist on cortically induced rhythmical jaw movements in the anesthetized guinea pig. Brain Res. 334: 201-206.

Chandler, S. H., L. J. Goldberg, and R. W. Lambert (1985b) The effects of orofacial sensory input on spontaneously occurring and apomorphine-induced rhythmical jaw movements in the anesthetized guinea pig. Neurosci. Lett. 53: 45-49.

Di Chiara, G., M. Morelli, A. Imperato, and M. L. Porceddu (1978) Substantia nigra as an efferent station for dopaminergic behavioural syndromes arising in the striatum. In Apomorphine and Other Dopaminomimetics, Vol. 1, G. L. Gessa and G. U. Corsini, eds., pp. 41-64, Raven, New York.

Dostrovsky, J. O., J. W. Hu, B. J. Sessle, and R. Sumino (1982) Stimulation sites in periaqueductal gray, nucleus raphe magnus and adjacent regions effective in suppressing oral-facial reflexes. Brain Res. 252: 287-297.

Drew, T., and S. Rossignol (1984) Phase-dependent responses evoked in limb muscles by stimulation of medullary reticular formation during locomotion in thalamic cats. J. Neurophysiol. 52: 653-675.

Dubner, R., B. J. Sessle, and A. T. Storey (1978) The Neural Basis of Oral and Facial Function, Plenum, New York, p. 483.

Edwards, S. B. (1975) Autoradiographic studies of the projections of the midbrain reticular formation: Descending projections of nucleus cuneiformis. J. Comp. Neurol. 161: 341-358.

Feldman, J. L. (1986) Neurophysiology of breathing in mammals. In Handbook of Physiology, Vol. 4, Intrinsic Regulatory Systems, F. E. Bloom, ed., pp. 463-524, American Physiological Society, Bethesda, MD.

Gebhart, G. F., J. Sandkuhler, J. G. Thalhammer, and M. Zimmerman (1983) Inhibition of spinal nociceptive information by stimulation in midbrain of the cat is blocked by lidocaine microinjected in nucleus raphe magnus and medullary reticular formation. J. Neurophysiol. 50: 1446-1459.

Goldberg, L. J., S. H. Chandler, and M. Tal (1982) Relationship between jaw movements and trigeminal motoneuron membrane-potential fluctuations during cortically induced rhythmic jaw movements in the guinea pig. J. Neurophysiol. 48: 110-125.

Grillner, S. (1981) Control of locomotion in bipeds, tetrapods, and fish. In Handbook of Physiology, Section 1: The Nervous System. Vol. 2, J. M. Brookhart and V. B. Mountcastle, eds., Williams \& Wilkins, Baltimore.

Hayashi, H., R. Sumino, and B. J. Sessle (1984) Functional organization of trigeminal subnucleus interpolaris: Nociceptive and innocuous afferent inputs, projections to thalamus, cerebellum, and spinal cord, and descending modulation from periaqueductal gray. J. Neurophysiol. 51: 890-905.

Kirk, R. E. (1982) Experimental Design: Procedures for the Behavioral Sciences, Wadsworth, Belmont, CA.

Lambert, R. W., L. J. Goldberg, and S. H. Chandler (1985) The relationship between cortically induced mandibular movements and lateral pterygoid and digastric muscle EMG activity in the anesthetized guinea pig. Brain Res. 329: 7-17.

Lennard, P. (1985) Afferent perturbations during "monopodal" swimming movements in the turtle: Phase-dependent cutaneous modulation and proprioceptive resetting of the locomotor rhythm. J. Neurosci. 5: 1434-1445.

Luschei, E. S., and L. J. Goldberg (1981) Neural mechanisms of mandibular control. In Handbook of Physiology, Vol. 2, Motor Control, 
Part 2, J. M. Brookhart, V. B. Mountcastle, V. B. Brooks, and S. R. Geiger, eds., pp. 1237-1274, American Physiological Society, Bethesda, MD

Marini, G., and M. L. Sotgiu (1985) Single unit activity in lateral reticular nucleus during cortically evoked masticatory movements in rabbits. Brain Res. 337: 287-292.

Mizuno, N., Y. Yasui, S. Nomura, K. Itoh, A. Konishi, M. Takada, and M. Kudo (1983) A light and electron microscope study of premotor neurons for the trigeminal motor nucleus. J. Comp. Neurol 215: 290-298

Nakamura, Y., M. Takatori, S. Nozaki, and M. Kikuchi (1975) Monosynaptic reciprocal control of trigeminal motoneurons from the medial bulbar reticular formation. Brain Res. 89: 144-148.

Nakamura, Y., M. Takatori, Y. Kubo, S. Nozaki, and S. Enomoto (1979) Masticatory rhythm formation-facts and a hypothesis. In Integrative Control Functions of the Brain, Vol. 2, N. Tsukahara, $\mathrm{K}$ Kubots, and K. Yagi, eds., pp. 321-331, Kodansh Scientific, Tokyo.

Nakamura, Y., S. Enomoto, and M. Katoh (1980) The role of medial bulbar reticular neurons in the orbital cortically induced masticatory rhythm in cats. Brain Res. 202: 207-212.

Nakamura, Y., K. Hiraba, S. Enomoto, and Y. Sahara (1982) Bulbar reticular unit activity during food ingestion in the cat. Brain Res. 253 . 312-316.

Nakamura, Y., K. Hiraba, M. Taira, Y. Sahara, S. Enomoto, M. Katoh, and A. Iriki (1984) Activity during active sleep of bulbar reticular neurons firing rhythmically during mastication in cats. Exp. Neurol. 85: $178-186$.

Nozaki, S., S. Enomoto, and Y. Nakamura (1983) Identification and input-output properties of bulbar reticular neurons involved in the cerebral cortical control of trigeminal motoneurons in cats. Exp. Brain Res. 49: 363-372.

Nozaki, S., A. Iriki, and Y. Nakamura (1986) Localization of central rhythm generator involved in cortically induced rhythmical masticatory jaw-opening movement in the guinea pig. J. Neurophysiol. 55 . 806-825.

Oliveras, J. L., A. Woda, G. Guilbaud, and J. M. Besson (1974) Inhibition of the jaw opening reflex by electrical stimulation of the periaqueductal gray matter in the awake, unrestrained cat. Brain Res. 72: 328-331.

Orlovsky, G. N. (1972) The effect of different descending systems on flexor and extensor activity during locomotion. Brain Res. 40:359371.

Petrovicky, P. (1966) A comparative study of the reticular formation of the guinea. J. Comp. Neurol. 123: 85-107.

Ranck, J. B. (1975) Which elements are excited in electrical stimulation of mammalian central nervous system: A review. Brain Res. 98: 417-440.

Redgrave, P., P. Dean, T. P. Donohoe, and S. G. Pope (1980) Superior colliculus lesions selectively attenuate apomorphine-induced oral stereotypy: A possible role for the nigro-tectal pathway. Brain Res. 196: 541-546.

Robinson, D. A. (1981) Control of eye movements. In Handbook of Physiology, Vol. 2, Motor Control, Part 2, J. M. Brookhart, V. B. Mountcastle, V. B. Brooks, and S. R. Geiger, eds., pp. 1275-1320, American Physiological Society, Bethesda, MD.

Rossner, W. (1965) Stereotaktischer Hirnatlas vom Meerschweinchen, Palla, Lochham bei Munchen.

Russell, D. F., and F. E. Zajac (1979) Effects of stimulating Deiters' nucleus and medial longitudinal fasciculus on the timing of the fictive locomotor rhythm induced in cats by DOPA. Brain Res. 177: 588592.

Sessle, B. J., and J. W. Hu (1981) Raphe-induced suppression of the jaw-opening reflex and single neurons in trigeminal subnucleus oralis, and influence of naloxone and subnucleus caudalis. Pain 10:19-36.

Sessle, B. J., J. W. Hu, R. Dubner, and G. E. Lucier (1981a) Functional properties of neurons in cat trigeminal subnucleus caudalis (medullary dorsal horn) II: Modulation of responses to noxious and nonnoxious stimuli by periaqueductal gray, nucleus raphe magnus, cerebral cortex and afferent influences, and effect of naloxone. J. Neurophysiol. 45: 193-207.

Sessle, B. J., G. J. Ball, and G. E. Lucier (1981b) Suppressive influences from periaqueductal gray and nucleus raphe magnus on respiration and related refiex activities and on solitary tract neurons, and effect of naloxone. Brain Res. 216: 145-161.

Siegal, J. M. (1979) Behavioral functions of the reticular formation. Brain Res. Rev. 1: 69-105.

Steriade, M., and J. A. Hobson (1976) Neuronal activity during the sleep-waking cycle. Prog. Neurobiol. 6: 155-376.

Sumi, T. (1971) Modification of cortically evoked rhythmic chewing and swallowing from midbrain and pons. Jpn. J. Physiol. 21: 489506.

Sumino, R. (1971) Central neural pathways involved in the jaw-opening reflex in the cat. In Oral-Facial Sensory and Motor Mechanisms, R. Dubner and Y. Kawamura, eds., pp. 315-329, Appleton-CenturyCrofts, New York.

Takatori, M., S. Nozaki, and Y. Nakamura (1981) Control of trigeminal motoneurons exerted from bulbar reticular formation in the cat. Exp. Neurol. 72: 122-140.

Travers, J. B., and R. Norgren (1983) Afferent projections to the oral motor nuclei in the rat. J. Comp. Neurol. 220: 280-298.

Von Euler, C. (1983) On the Origin and Pattern Control of Breathing Rhythmicity in Mammals, A. Roberts and B. L. Roberts, eds., pp. 469-485, University Press, Cambridge.

Vornov, J. J., and J. Sutin (1983) Brainstem projections to the normal and noradrenergically hyperinnervated trigeminal motor nucleus. $\mathbf{J}$. Comp. Neurol. 214: 198-208.

Wyman, R. J. (1977) Neural generation of the breathing rhythm. Annu. Rev. Physiol. 39: 417-448. 